\title{
The Casimir Effect in Topological Field Theory: Case of Elliptic Genera
}

\author{
A. A. Bytsenko \\ Departamento de Física, Universidade Estadual de Londrina, Caixa Postal 6001, \\ Londrina-Paraná, Brazil \\ E-mail: aabyts@gmail.com

\section{E. Elizalde} \\ Consejo Superior de Investigaciones Científicas, ICE/CSIC and IEEC, \\ Campus UAB, Facultat de Ciències, Torre C5-Par-2pl, 08193 Bellaterra (Barcelona) Spain \\ E-mail: elizalde@ieec.uab.es, elizalde@math.mit.edu
}

\section{R. M. Luna}

Departamento de Física, Universidade Estadual de Londrina, Caixa Postal 6001,

Londrina-Paraná, Brazil

E-mail: luna@uel.br

\begin{abstract}
The coupling between the quantum generating functions of a field theory and corresponding formal power series, associated with dimensions of chains and homologies of suitable Lie algebras, is explored. This interrelation is further analyzed in view to its application to the Casimir effect for partition functions of minimal three-dimensional gravities, in space-times which are asymptotic to, respectively, $A d S_{3}$, pure $N=1$ supergravity, two-variable elliptic genera of an hypersurface, and complete intersections. All these examples are here viewed as straightforward applications of the machinery of modular forms and spectral functions to the one-loop contributions of quantum partition functions (e.g. the Casimir effect) which are represented by means of formal power series.
\end{abstract}

7th Conference Mathematical Methods in Physics - Londrina 2012,

16 to 20 April 2012

Rio de Janeiro, Brazil 


\section{Introduction}

We deal in this paper with applications of modular forms (and spectral functions related to the congruence subgroup of $S L(2, \mathbb{Z})$ ) to partition functions connected to suitable Lie algebras. The connection that can be established is particularly striking in the case of the correspondence between three-dimensional quantum gravity, in a space-time which is asymptotic to $A d S_{3}$, and $2 D$ conformal field theories. In the case of $A d S_{3}$ one has Selberg-type spectral functions and Ruelle functions while, on the field theory side, the correspondence is established with the Casimir effect for quantum contribution to the partition functions. We seek appropriate expressions for the partition functions of minimal three-dimensional gravities in a space-time which is asymptotic to, respectively, $A d S_{3}$, pure $N=1$ supergravity, two-variable elliptic genera of an hyperesurface, and complete intersections. In the case of hypersurfaces the elliptic genera coincide with those of the orbifold Landau-Ginzburg model [1].

Elliptic genera are natural topological invariants, which generalize classical genera. They appear, for instance, when one considers the supersymmetric indices of superconformal vertex algebras. For mathematicians elliptic genera (and the respective elliptic cohomology) may be associated to new mathematical invariants for spaces while, for physicists, elliptic genera are one-loop string partition functions and, therefore, they are very relevant in topological Casimir effect calculations [2,3]. They have been proven to be useful in black hole entropy computations, too [4].

Let us briefly explain the construction of the action of the Heisenberg/Clifford algebra on homology groups of varieties, which is key to this project. One can start with the construction of (integrable) highest weight representation of the affine Lie algebra on the homology group of moduli spaces of torsion-free sheaves. Thus the generators of the affine Lie algebra (as a KacMoody algebra) are given by moduli spaces of sheaves. Then, the characters of highest weight modules may be identified with the holomorphic parts of partition functions on the torus, for the corresponding field theories. This structures arises naturally, but not exclusively, in string theory, and is particularly clear and treatable when supersymmetry is involved.

Our interest in this example stems from the $A d S_{3} / C F T_{2}$ correspondence. The geometric structure of three-dimensional gravity (and black holes) allows for exact computations, since its Euclidean counterpart is locally isomorphic to a constant curvature hyperbolic space. There is an agreement between spectral functions related to Euclidean $A d S_{3}$ and modular-like functions (Poincare series). This occurs when the arguments of the spectral functions take values on a Riemann surface, viewed as the conformal boundary of $A d S_{3}$. According to the holographic principle, a strong correspondence exists between certain field theory quantities in the bulk of an $A d S_{3}$ manifold and related quantities on its boundary at infinity. To be more precise, the classes of Euclidean $A d S_{3}$ spaces are quotients of the real hyperbolic space by a discrete group (a Schottky group). The boundaries of these spaces can be oriented compact surfaces with a conformal structure (compact complex algebra ic curves).

In Sect. 2 we consider multiplicative operations on graded vector spaces which are useful for the two-variable elliptic genus. We introduce the Petterson-Selberg and Ruelle spectral functions of hyperbolic three-geometry.

In Sect. 3 we discuss the theories of a minimal three-dimensional quantum gravity in a spacetime asymptotic to $A d S_{3}$, and the Neveu-Schwarz and Ramond sectors of $N=1$ supergravity. The 
symmetry group of $A d S_{3}$ gravity (with appropriate boundary conditions) is generated by the Virasoro algebra, and the one-loop partition function is indeed the partition function of a conformal field theory in two dimensions. We investigate the corresponding Casimir effect, namely one-loop corrections to the three-dimensional gravity: the holomorphic contribution to the partition functions corresponds to the formal character of the Virasoro-module [5]. We extend our results to $N=1$ supergravity and show there that the infinite series of quantum corrections for the cases of three-dimensional gravity and the Neveu-Schwarz and Ramond sectors of supergravity can be actually reproduced in terms of spectral functions of Selberg type in a holomorphically factorized theory. Three-dimensional gravity (as a function of the inverse temperature, $\beta$ ) exhibits, in the semi-classical limit, a Hawking-Page phase transition for a thermal gas of Brown-Henneaux boundary excitations. The analysis of the condensation of Lee-Yang zeros of the partition function on the phase boundary clearly shows that this phase transition can be compatible with holomorphic factorization [6]. We assume that, in accordance with the holographic principle, the zeros of the partition function are encoded in an analytic structure of Selberg-type meromorphic functions.

In Sect. 4 we briefly consider two-variable elliptic genera for a complex manifold, in particular, the elliptic genus of a complex manifold, the Hirzebruch $\chi_{y}$-genus, the Riemann-Roch number of a holomorphic vector bundle on a complex manifold, and the arithmetic genus of an irreducible projective curve.

In Sect. 5 we discuss and calculate-in terms of spectral functions of the hyperbolic threegeometry - the elliptic genera of an hypersurface and of a smooth complete intersection and make manifest the similarities between the two cases. Finally, in Sect. 6 we provide some conclusions.

\section{Prototypes for the elliptic genera}

The problem of defining elliptic genera for singular varieties has been addressed in [?], where the definitions for orbifolds and for pairs consisting of projective varieties are given. The authors of that work used resolutions of the singularities and proved their definitions to be independent of these resolutions. In the present article we follow a completely different approach. More precisely, we will use resolutions of the structure sheaf, instead of the variety itself.

For a holomorphic vector bundle, $E$ on $X$, and a formal variable, $z$, we use the following identities

$$
\begin{aligned}
S_{q}(z E) & =1 \oplus z q E \oplus z^{2} q^{2} \operatorname{Sym}^{2} E \oplus z^{3} q^{3} \operatorname{Sym}^{3} E \oplus \cdots=S_{z q} E, \\
\Lambda_{q}(z E) & =1 \oplus z q E \oplus z^{2} q^{2} \operatorname{Alt}^{2} E \oplus z^{3} q^{3} \operatorname{Alt}^{3} E \oplus \cdots=\Lambda_{z q} E, \\
S_{q}(z E)^{\mathbb{C}} & =S_{q}(z E) \otimes S_{q}(\bar{z} \bar{E}), \\
\Lambda_{q}(z E)^{\mathbb{C}} & =\Lambda_{q}(z E) \otimes \Lambda_{q}(\bar{z} \bar{E}) .
\end{aligned}
$$

These identities have good multiplicative properties and its elements should be understood as elements of the $K$-theory of the underlying space.

$$
\begin{aligned}
S_{q}(E \oplus F) & =\left(S_{q} E\right) \otimes\left(S_{q} F\right), \quad S_{q}(E \ominus F)=\left(S_{q} E\right) \otimes\left(S_{q} F\right)^{-1}, \\
\Lambda_{q}(E \oplus F) & =\left(\Lambda_{q} E\right) \otimes\left(\Lambda_{q} F\right), \quad \Lambda_{q}(E \ominus F)=\left(\Lambda_{q} E\right) \otimes\left(\Lambda_{q} F\right)^{-1} .
\end{aligned}
$$


In Eqs. (2.5) and (2.6) we have used the fact that

$$
\begin{aligned}
\operatorname{Sym}^{n}(E \oplus F) & =\bigoplus_{i=0}^{n} \operatorname{Sym}^{i}(E) \otimes \operatorname{Sym}^{n-i}(F), \\
\operatorname{Alt}^{n}(E \oplus F) & =\bigoplus_{i=0}^{n} \operatorname{Alt}^{i}(E) \otimes \operatorname{Alt}^{n-i}(F) .
\end{aligned}
$$

In the case of a line bundle $\mathscr{L}$, we have $S_{q} \mathscr{L}=1 \bigoplus_{n \in \mathbb{Z}_{+}} q^{n} \mathscr{L}^{n}=(1 \ominus q \mathscr{L})^{-1}=\left(\Lambda_{-q} \mathscr{L}\right)^{-1}$, and therefore $\left(S_{q} E\right)^{-1}=\Lambda_{-q} E$ for any vector bundle $E$, and similarly $\left(\Lambda_{q} E\right)^{-1}=S_{-q} E$. The prototypes for the elliptic genera are the expressions (see for detail [7]):

$$
\begin{array}{ll}
\bigotimes_{n \in \mathbb{Z}_{+}} S_{\sigma q^{n}}\left((\xi \mathscr{P})^{\mathbb{C}}\right) \bigotimes_{n \in \mathbb{Z}_{+}} \Lambda_{\lambda q^{n}}\left((\zeta \mathscr{Q})^{\mathbb{C}}\right), & \bigotimes_{n \in \mathbb{Z}_{+}} S_{\sigma q^{n}}\left((\xi \mathscr{P})^{\mathbb{C}}\right) \bigotimes_{n \in \mathbb{Z}_{+} / 2} \Lambda_{\lambda q^{n}}\left((\zeta \mathscr{Q})^{\mathbb{C}}\right),(2.9) \\
\bigotimes_{n \in \mathbb{Z}_{+} / 2} S_{\sigma q^{n}}\left((\xi \mathscr{P})^{\mathbb{C}}\right) \bigotimes_{n \in \mathbb{Z}_{+}} \Lambda_{\lambda q^{n}}\left((\zeta \mathscr{Q})^{\mathbb{C}}\right), & \bigotimes_{n \in \mathbb{Z}_{+} / 2} S_{\sigma q^{n}}\left((\xi \mathscr{P})^{\mathbb{C}}\right) \bigotimes_{n \in \mathbb{Z}_{+} / 2} \Lambda_{\lambda q^{n}}\left((\zeta \mathscr{Q})^{\mathbb{C}}\right)(2
\end{array}
$$

Let us recall some well known examples of vertex operator algebra bundles, which have been used in the literature to study the elliptic genus and the Witten genus. If $X$ is a Riemannian manifold, then the transition functions of the complex tangent bundle $(T X)^{\mathbb{C}}$ lie in the special orthogonal group $S O(d)$, where $d$ is the dimension of $X$. Then $\bigotimes_{n \in \mathbb{Z}_{+}} S_{q^{n}}\left((T X)^{\mathbb{C}}\right)$ is a $V_{H}^{S O(d)}$-bundle. Here $V_{H}$ is the Heisenberg vertex operator algebra of dimension $d$, with $S O(d)$ as a subgroup of $\operatorname{Aut}\left(V_{H}\right)$, and $V_{H} S O(d)$ is the set of $S O(d)$-invariants of $V_{H}$, which is the vertex operator subalgebra of $V_{H}$. Similarly, $\bigotimes_{n \in \mathbb{Z}_{+} \cup\{0\}} \Lambda_{q^{n+1 / 2}}\left(T_{\mathbb{C}} X\right)$ is an $L(1,0)^{S O(d)}$-bundle, where $L(1,0)$ is the level one module for the affine algebra $D_{d / 2}^{(1)}$. In this case we assume that $d$ is even. If $X$ is further assumed to be a spin manifold, we denote the spin bundle by $\mathscr{S}$. Then $\mathscr{S} \otimes \bigotimes_{n \in \mathbb{Z}_{+}} \Lambda_{q^{n}}\left((T X)^{\mathbb{C}}\right)$ is also a $L(1,0)^{S O(d)}$-bundle.

For $\bigotimes_{n \in \mathbb{Z}_{+}} S_{q^{n}}\left((T X)^{\mathbb{C}}\right)$ the resulting Chern character takes the form

$$
\begin{aligned}
\operatorname{ch}\left(\bigotimes_{n \in \mathbb{Z}_{+}} S_{q^{n}}\left((T X)^{\mathbb{C}}\right)\right) & =\prod_{j} \prod_{n \in \mathbb{Z}_{+}}\left[\left(1-q^{n} e^{x_{j}}\right)\left(1-q^{n} e^{-x_{j}}\right)\right]^{-1} \\
& =\prod_{j}\left[\mathscr{R}\left(s=\widehat{x}_{j}(1-i t)\right) \cdot \mathscr{R}\left(s=-\widehat{x}_{j}(1-i t)\right)\right]^{-1},
\end{aligned}
$$

where $q=\exp (2 \pi i \tau), t=\operatorname{Re} \tau / \operatorname{Im} \tau$ and $\widehat{x}_{j}=x_{j} / 2 \pi i$. One of the important feature of the theory of infinite dimensional Lie algebras is the modular properties of characters of certain representations. The Chern polynomials (and elliptic genera) can be converted into product expressions which inherit modular and cohomological properties (in the sense of characteristic classes of foliations) of appropriate (polygraded) Lie algebras. The final result can be written in terms of spectral functions of the hyperbolic three-geometry associated with $q$-series. The spectral Patterson-Selberg and Ruelle functions, $Z_{\Gamma}(s)$ and $\mathscr{R}(s)$ respectively, can be attached to a closed oriented hyperbolic three-manifold $X=H^{3} / \Gamma$ (with an acyclic orthogonal representation of $\pi_{1}(X)$ ), as follows [8, 7]:

$$
\begin{aligned}
& Z_{\Gamma}(s):=\prod_{k_{1}, k_{2} \in \mathbb{Z}_{+} \cup\{0\}}\left[1-\left(e^{i \beta}\right)^{k_{1}}\left(e^{-i \beta}\right)^{k_{2}} e^{-\left(k_{1}+k_{2}+s\right) \alpha}\right], \quad \mathscr{R}(s)=\prod_{p=0}^{\operatorname{dim} X-1} Z_{\Gamma}(p+s)^{(-1)^{p}}, \\
& \prod_{n=\ell}^{\infty}\left(1-q^{n+\varepsilon}\right)=\mathscr{R}(s=\xi(1-i t)) \quad(\xi=\ell+\varepsilon) .
\end{aligned}
$$




\section{The Casimir effect in three-dimensional gravity and $N=1$ supergravity}

A remarkable link between the theory of the highest-weight modules over the Virasoro algebra, conformal field theory, and statistical mechanics was discovered in $[9,10]$. Here we briefly note some elements of the representation theory of the Virasoro algebra which are, in fact, very similar to those for Kac-Moody algebras. Consider the highest-weight representation of the Virasoro algebra. Let $M(c, h)(c, h \in \mathbb{C})$ be the Verma module over the Virasoro algebra (see, for example, [11]). The conformal central charge $c$ acts on $M(c, h)$ as $c I$. As $\left[\mathbf{e}_{0}, \mathbf{e}_{-j}\right]=n \mathbf{e}_{-j}$, $\mathbf{e}_{0}$ is diagonalizable on $M(c, h)$, with spectrum $h+\mathbb{Z}_{+}$and eigenspace decomposition given by: $M(c, h)=\bigoplus_{j \in \mathbb{Z}_{+}} M(c, h)_{h+j}$, where $M(c, h)_{h+j}$ is spanned by elements of the basis $\left\{\mathbf{e}_{-j_{k}}\right\}_{k=1}^{n}$ of $M(c, h)$. The number $Z_{j}=\operatorname{dim} M(c, h)_{h+j}$, is the classical partition function. This means that the Konstant partition function for the Virasoro algebra is in fact the classical partition function. On the other hand, the partition functions can be rewritten in the form

$$
\operatorname{Tr}_{M(c, h)} q^{\mathbf{e}_{0}}:=\sum_{\lambda} \operatorname{dim} M(c, h)_{\lambda} q^{\lambda}=q^{h} \prod_{j=1}^{\infty}\left(1-q^{j}\right)^{-1} .
$$

The series $\operatorname{Tr}_{\mathscr{V}} q^{\mathbf{e}_{0}}$ is called the formal character of the Virasoro-module $\mathscr{V}$.

Quantum gravity in $A d S_{3}$. It has been shown that the contribution to the partition function of three-dimensional gravity in a space-time asymptotic to $A d S_{3}$ comes from smooth geometries $X=A d S_{3} / \Gamma$, where $\Gamma$ is a discrete subgroup of $S O(3,1)$. To be more precise, it comes from geometries $X_{c, d}$ (see for detail [6]), where $c$ and $d$ are a pair of relatively prime integers, $c \geq 0$, and a pair $(c, d)$ is identified with $(-c,-d)$. The manifolds $X_{c, d}$ are all diffeomorphic to each other, and therefore the contribution $W_{c, d}(\tau)$ to the partition function can be expressed in terms of any one of them, say $W_{0,1}(\tau)$, by a modular transformation. One has the following formula

$$
W_{c, d}(\tau, \bar{\tau})=W_{0,1}((a \tau+b) /(c \tau+d), \bar{\tau}), \quad W_{0,1}(\tau, \bar{\tau}):=|q \bar{q}|^{-k} \prod_{n=2}^{\infty}\left|1-q^{n}\right|^{-2} .
$$

In Eq. (3.2) $24 k=c_{L}=c_{R}=c$, and $c$ is the central charge of a conformal field theory, $q=$ $\exp (2 \pi i \tau)=\exp [2 \pi(-\operatorname{Im} \tau+i \operatorname{Re} \tau)]$ such that $|q \bar{q}|^{-k}=\exp (4 \pi k \operatorname{Im} \tau)$ corresponds to the classical prefactor.

Recall that $H^{3} / \Gamma$ is also the geometry of a Euclidean three-dimensional black hole. To make a correspondence between models one must set $k=(8 \pi G)^{-1}$.

$$
-\log W_{0,1(\text { classical })}(\tau, \bar{\tau})=k \log |\bar{q} q|=2 \pi r_{+}(4 G)^{-1},
$$

where $r_{+}>0$ is the outer horizon of a black hole. This result is the classical part of the contribution; Eq. (3.2) is one-loop exact as has been claimed in [6]. Note that the one-loop contribution (3.2) is qualitatively similar to the quantum correction to a three-dimensional black hole $[12,13]$. On general grounds one would expect that the generating function (3.2) would be connected to the relevant one-loop determinant. Indeed, one-loop determinants can be re-summed with the help of the Poisson summation procedure and may give a possibility to realize this connection. (An analogous procedure of Poisson summation for regularized Poincaré series associated with $W(\tau)$ has been analyzed in [6], Sect. 3.2.). 
Holomorphic factorization of supergravity quantum corrections. In this subsection we show that quantum corrections for three-dimensional gravity and for the Neveu-Schwarz and Ramond sector of $N=1$ supergravity can be reproduced in terms of Selberg-type spectral functions in a holomorphically factorized theory. For three-dimensional gravity in a real hyperbolic space the one-loop generating function has the form of a product of holomorphic and antiholomorphic functions. The full gravity partition function (3.2) admits the factorization $W_{0,1}(\tau, \bar{\tau})=$ $W(\tau)_{\text {hol }} \cdot W(\bar{\tau})_{\text {antihol }}$, where

$$
W(\tau)_{\mathrm{hol}}=q^{-k} \prod_{n=1}^{\infty}\left(1-q^{n+1}\right)^{-1}, \quad W(\bar{\tau})_{\mathrm{antihol}}=\bar{q}^{-k} \prod_{n=1}^{\infty}\left(1-\bar{q}^{n+1}\right)^{-1} .
$$

Note that the holomorphic contribution in (3.4) corresponds to the formal character of the Virasoromodule (3.1).

The modulus of a Riemann surface $\Sigma$ of genus one (the conformal boundary of $A d S_{3}$ ) is defined up to $\gamma \cdot \tau=(a \tau+b) /(c \tau+d)$ with $\gamma \in S L(2, \mathbb{Z})$. The generating function as the sum of known contributions of states of left- and right-moving modes in the conformal field theory takes the form

$$
\sum_{c, d} W_{c, d}(\tau, \bar{\tau})=\sum_{c, d} W_{0,1}((a \tau+b) /(c \tau+d), \bar{\tau})
$$

We would like to comment about the sum over geometries. The generating function, including the contribution coming from the Brown-Henneaux excitations, have the form

$$
\begin{aligned}
\sum_{c, d} W_{c, d}(\gamma \cdot \tau, \bar{\tau}) & =\sum_{c, d}\left|q^{-k} \prod_{n=2}^{\infty}\left(1-q^{n}\right)^{-1}\right|_{\gamma}^{2} \\
& =\sum_{c, d}\left\{|q \bar{q}|^{-k} \cdot[\mathscr{R}(s=2-2 i t)]_{\mathrm{hol}}^{-1} \cdot[\mathscr{R}(\bar{s}=2+2 i t)]_{\mathrm{antihol}}^{-1}\right\}_{\gamma}
\end{aligned}
$$

Here $|\ldots|_{\gamma}$ denotes the transform of an expression $|\ldots|$ by $\gamma$. The summand in (3.6) is independent of the choice of $a$ and $b$ in $\gamma$. Note that the sum over $c$ and $d$ in (3.6) should be thought of as a sum over the coset $P S L(2, \mathbb{Z}) / \mathbb{Z} \equiv(S L(2, \mathbb{Z}) /\{ \pm 1\}) / \mathbb{Z}$. Three-dimensional gravity (as a function of the inverse temperature, $\beta$ ), exhibits in the semi-classical limit a Hawking-Page phase transition for a thermal gas of Brown-Henneaux boundary excitations. The analysis of the condensation of Lee-Yang zeros of the partition function on the phase boundary, shows that this phase transition can be compatible with holomorphic factorization [6]. We assume that, in accordance with the holography principle, the zeros of the partition function are encoded in the analytic structure of the Selberg-type meromorphic functions.

The Neveu-Schwarz and Ramond sectors of $N=1$ supergravity. We shall consider only the basic case of $N=1$ supergravity (see [6] for an explanation). The symmetry group $S L(2, \mathbb{R}) \times$ $\operatorname{SL}(2, \mathbb{R})$ of $A d S_{3}$ is replaced by $\operatorname{OSp}(1 \mid 2) \times \operatorname{OSp}(1 \mid 2)$, where $\operatorname{OSp}(1 \mid 2)$ is a supergroup whose bosonic part is $\operatorname{Sp}(2, \mathbb{R})=S L(2, \mathbb{R})$. The boundary CFT has $(1,1)$ supersymmetry $(N=1$ supersymmetry for both left- and right-movers). There are a few closely related possible choices for the partition function: $\operatorname{Tr} \exp (-\beta H-i \theta J)$ or $\operatorname{Tr}(-1)^{F} \exp (-\beta H-i \theta J)$. The conserved angular momentum $J=L_{0}-\bar{L}_{0}$ generates a rotation at infinity of the asymptotic $A d S_{3}$ space-time. The operator $(-1)^{F}$ is equivalent to $(-1)^{2 J}$; states of integer or half-integer $J$ are bosonic or fermionic, 
respectively. This property is inherited from the perturbative spectrum of Brown-Henneaux excitations. The trace could be computed in either the Neveu-Schwarz (NS) or the Ramond (R) sector. One can compute these partition functions by summing over three-manifolds $X$ that are locally $A d S_{3}$ and whose conformal boundary is a Riemann surface $\Sigma$ of genus one. The four possible partition functions associated with NS or R sectors (with or without an insertion of $\left.(-1)^{F}\right)$ correspond to the four spin structures on $\Sigma$. An element $g$ of $G=S L(2, \mathbb{R})$ acts on a spin structure by $g \cdot\left[\begin{array}{l}\mu \\ v\end{array}\right] \rightarrow\left[\begin{array}{ll}a & b \\ c & d\end{array}\right] \cdot\left[\begin{array}{l}\mu \\ v\end{array}\right], \mu, v \in(1 / 2) \mathbb{Z} / \mathbb{Z}$, where the four spin structures on the two-torus $\Sigma$ are represented by the column vector, and $\mu, v$ take the values (1/2) for antiperiodic (NS) boundary conditions and 0 for periodic $(\mathrm{R})$ ones. Taking into account the choice of the spin structure on $\Sigma$, one can sum over choices of $X$ such that the given spin structure on $\Sigma$ does extend over $X$. The NS spin structure on $\Sigma$ is compatible with $X_{0,1}$, and therefore $X_{0,1}$ contributes to traces in the NS sector, and not to the $\mathrm{R}$ sector. The partition function of left- and right-moving excitations is $F(q, \bar{q})=\operatorname{Tr}_{\mathrm{NS}} \exp (-\beta H-i \theta J)$. Let us also study partition functions with other spin structures. If we let $\mu=0, v=1 / 2$, then we get $G(q, \bar{q})=\operatorname{Tr}_{\mathrm{NS}}(-1)^{F} \exp (-\beta H-i \theta J)$. Thus, the contribution to $F(q, \bar{q})$ and $G(q, \bar{q})$ associated with $X_{0,1}$, for all spin structures, becomes [6]:

$$
\begin{aligned}
& F_{0,1}(\tau, \bar{\tau})=F_{0,1}^{\text {(ground) }} \cdot \widehat{F}_{0,1}(\tau, \bar{\tau}) \equiv\left|q^{-k^{*} / 2}\right|^{2} \cdot\left|\prod_{n=2}^{\infty} \frac{1+q^{n-1 / 2}}{1-q^{n}}\right|^{2}, \\
& G_{0,1}(\tau, \bar{\tau})=G_{0,1}^{\text {(ground) }} \cdot \widehat{G}_{0,1}(\tau, \bar{\tau}) \equiv\left|q^{-k^{*} / 2}\right|^{2} \cdot\left|\prod_{n=2}^{\infty} \frac{1-q^{n-1 / 2}}{1-q^{n}}\right|^{2} .
\end{aligned}
$$

Here the contributions $F_{0,1}^{\text {(ground) }}=G_{0,1}^{\text {(ground) }} \equiv\left|q^{-k^{*} / 2}\right|^{2}$ are related to the groundstate energy; the contribution $G_{0,1}$ of $X_{0,1}$ is obtained by reversing the sign of all fermionic contributions in (3.7). The complete functions $F(\tau), G(\tau)$ can be computed by adding $F_{0,1}$ and $G_{0,1}$ over modular images with $(c+d)$ odd. It corresponds to the spin structure with $\mu=v=1 / 2$ and $\mu=0$ and $v=1 / 2$, respectively:

$$
\begin{aligned}
& \widehat{F}\left[\begin{array}{c}
\frac{1}{2} \\
\frac{1}{2}
\end{array}\right](\tau, \bar{\tau})=\sum_{c, d \mid(c+d) \text { odd }} \widehat{F}_{0,1}((a \tau+b) /(c \tau+d), \bar{\tau}), \\
& \widehat{G}\left[\begin{array}{c}
0 \\
\frac{1}{2}
\end{array}\right](\tau, \bar{\tau})=\sum_{c, d \mid d \text { odd }} \widehat{G}_{0,1}((a \tau+b) /(c \tau+d), \bar{\tau}) .
\end{aligned}
$$

Note that the summand in (3.9) and (3.10) does not depend on the choice of $a, b$. A modular transformation $\tau \rightarrow \tau+1$ exchanges the pair $(\mu, v)=(0,1 / 2)$ with $(\mu, v)=(1 / 2,1 / 2)$; in particular, $F(\tau)=G(\tau+1)=F(\tau+2)$. One can compute the Ramond partition function $K=$ $\operatorname{Tr}_{\mathrm{R}} \exp (-\beta H-i \theta J)$ for $\mu=1 / 2, v=0$, so $K(\tau)=G(-1 / \tau)$. This completes the list of three of the four partition functions. In a supersymmetric theory with discrete spectrum, the fourth partition function $Q=\operatorname{Tr}_{\mathrm{R}}(-1)^{F} \exp (-\beta H-i \theta J)$ is an integer, independent of $\beta$ and $\theta$ (it can be interpreted as the index of a supersymmetry generator). This function has to be computed using the odd spin structure, the one with $\mu=v=0$. Typically in three-dimensional gravity the partition function $Q$ vanishes, since the odd spin structure does not extend over any three-manifold with 
boundary $\Sigma$. Our goal here will be to use the spectral functions representation for holomorphycally $\{\mathscr{R}(s), \mathscr{R}(\sigma)\}$ and antiholomorphycally $\{\mathscr{R}(\bar{s}), \mathscr{R}(\bar{\sigma})\}$ factorized theory. We get (see also [5])

$$
\begin{aligned}
\widehat{F}_{0,1}(\tau, \bar{\tau}) & =\left[\frac{\prod_{n=2}^{\infty}\left(1+q^{n-1 / 2}\right)}{\prod_{n=2}^{\infty}\left(1-q^{n}\right)}\right] \cdot\left[\frac{\prod_{n=2}^{\infty}\left(1+\bar{q}^{n-1 / 2}\right)}{\prod_{n=2}^{\infty}\left(1-\bar{q}^{n}\right)}\right] \\
& =\left[\frac{\mathscr{R}(\sigma=3 / 2-(3 / 2) i t+i \eta(\tau))}{\mathscr{R}(s=2-2 i t)}\right] \cdot\left[\frac{\mathscr{R}(\bar{\sigma}=3 / 2+(3 / 2) i t+i \eta(\tau))}{\mathscr{R}(\bar{s}=2+2 i t)}\right], \\
\widehat{G}_{0,1}(\tau, \bar{\tau}) & =\left[\frac{\prod_{n=2}^{\infty}\left(1-q^{n-1 / 2}\right)}{\prod_{n=2}^{\infty}\left(1-q^{n}\right)}\right] \cdot\left[\frac{\prod_{n=2}^{\infty}\left(1-\bar{q}^{n-1 / 2}\right)}{\prod_{n=2}^{\infty}\left(1-\bar{q}^{n}\right)}\right] \\
& =\left[\frac{\mathscr{R}(s=3 / 2-(3 / 2) i t)}{\mathscr{R}(s=2-2 i t)}\right] \cdot\left[\frac{\mathscr{R}(\bar{s}=3 / 2+(3 / 2) i t)}{\mathscr{R}(\bar{s}=2+2 i t)}\right] .
\end{aligned}
$$

The complete functions $F(\tau)$ and $G(\tau)$ become

$$
\begin{aligned}
F\left[\begin{array}{c}
\frac{1}{2} \\
\frac{1}{2}
\end{array}\right](\tau, \bar{\tau}) & =\sum_{c, d \mid(c+d) \text { odd }} F_{0,1}^{\text {(ground })}(\gamma \cdot \tau, \bar{\tau}) \widehat{F}_{0,1}(\gamma \cdot \tau, \bar{\tau}) \\
& =\sum_{c, d \mid(c+d) \text { odd }} F_{0,1}^{\text {(ground })}(\gamma \cdot \tau, \bar{\tau})\left\{\left[\frac{\mathscr{R}(\sigma=3 / 2-(3 / 2) i t+(1+2 d / c) i \eta(\tau))}{\mathscr{R}(s=2-2 i t+i \eta(\tau))}\right]\right\}_{\gamma} \\
& \times\left\{\left[\frac{\mathscr{R}(\bar{\sigma}=3 / 2+(3 / 2) i t+(1+2 d / c) i \eta(\tau))}{\mathscr{R}(\bar{s}=2+2 i t+i \eta(\tau))}\right]\right\}_{\gamma} \\
G\left[\begin{array}{c}
0 \\
\frac{1}{2}
\end{array}\right](\tau, \bar{\tau})= & \sum_{c, d \mid(c+d) \text { odd }} G_{0,1}^{\text {(ground })}(\gamma \cdot \tau, \bar{\tau}) \widehat{G}_{0,1}(\gamma \cdot \tau, \bar{\tau}) \\
= & \sum_{c, d \mid(c+d) \text { odd }} G_{0,1}^{\text {(ground })}(\gamma \cdot \tau, \bar{\tau})\left\{\left[\frac{\mathscr{R}(s=3 / 2-(3 / 2) i t+(1+2 d / c) i \eta(\tau))}{\mathscr{R}(s=2-2 i t+(2 d / c) i \eta(\tau))}\right]\right\}_{\gamma} \\
& \times\left\{\left[\frac{\mathscr{R}(\bar{s}=3 / 2+(3 / 2) i t+(1+2 d / c) i \eta(\tau))}{\mathscr{R}(\bar{s}=2+2 i t+(2 d / c) i \eta(\tau))}\right]\right\}_{\gamma}
\end{aligned}
$$

In the case the final sums (3.6), (5.5), and (3.14) are divergent (this kind of divergences have also been encountered in similar sums in $[14,15,16])$ the one-loop corrections has to be regularized. This procedure can be developed by using the Poisson summation technique in a way similar to the calculations of Poincaré series in [6], Sect. 3.2 (see also [17]). This is a natural regularization which can actually be viewed as just a type of the zeta function regularization, as proven in many related examples $[2,18]$. We hope we will return to this interesting problem in a forthcoming paper.

\section{Two-variable elliptic genera for a complex manifold}

In the physics literature a two-variable elliptic genus can be associate with $N=(2,2)$ superconformal field theory (cf. [19, 20, 21]). A two-variable elliptic genus is given by

$$
\operatorname{Tr}_{\mathscr{H}}(-1)^{F} y^{J_{0}} q^{L_{0}-c / 24} \bar{q}^{\bar{L}_{0}-c / 24}
$$


where $\mathscr{H}$ is the Hilbert space of the superconformal field theory, $L_{0}$ (resp. $\bar{L}_{0}$ ) is the Virasoro generator of left (resp. right)-movers and $J_{0}\left(\operatorname{resp} \bar{J}_{0}\right)$ is the $U(1)$ charge operator of left (resp. right)-movers. In addition the trace is taken over the Ramond sector and $F=F_{L}-F_{R}$ with $F_{L}$ (resp. $F_{R}$ ) the fermion number of left (resp. right)-movers. Let $X$ be a smooth manifold, one has the following mathematical expression for the field theory genus (cf. [21, 22, 23]):

$$
\operatorname{Ell}(X)=\int_{X} \operatorname{ch}(\mathscr{E} l l(q, y)) \operatorname{Td}(X),
$$

where

$$
\mathscr{E} l l(T X ; q, y)=y^{-\operatorname{dim} X / 2} \bigotimes_{n \in \mathbb{Z}_{+}}\left(\Lambda_{-y q^{n-1}}(\overline{T X}) \otimes \Lambda_{-y^{-1} q^{n}} T X \otimes S_{q}(\overline{T X}) \otimes S_{q^{n}} T X\right) .
$$

- Suppose $X \equiv X^{\mathbb{C}}$ is a compact complex manifold of dimension $d$. Let $T X$ denote the holomorphic tangent bundle of $X$, and consider the holomorphic vector bundle $\mathscr{E} l l(T X ; q, y)$. The formal power series

$$
\begin{aligned}
\chi(X ; q, y) & :=\chi(X, \mathscr{E} l l(T X ; q, y)) \\
\stackrel{(\text { by HRR })}{=} & y^{-d / 2} \int_{X^{\mathbb{C}}} \prod_{j \in \mathbb{Z}_{+}}^{d} x_{j} \prod_{n \in \mathbb{Z}_{+}} \frac{\left(1-y q^{n-1} e^{-x_{j}}\right)\left(1-y^{-1} q^{n} e^{x_{j}}\right)}{\left(1-q^{n-1} e^{-x_{j}}\right)\left(1-q^{n} e^{x_{j}}\right)} \\
& =\left[\frac{\mathscr{R}\left(s=\left(w-\widehat{x}_{j}\right)(1-i t)\right)}{\mathscr{R}\left(s=-\widehat{x}_{j}(1-i t)\right)}\right]\left[\frac{\mathscr{R}\left(s=\left(1-w+\widehat{x}_{j}\right)(1-i t)\right)}{\mathscr{R}\left(s=\left(1+\widehat{x}_{j}\right)(1-i t)\right)}\right]
\end{aligned}
$$

is called the elliptic genus of $X$, and its computation (last line in Eq. (4.4)) follows from the Hirzebruch-Riemann-Roch (HRR) theorem. In Eq. (4.4) we put $y=\exp (2 \pi i w)$.

- For a compact complex $d$-manifold $X$, one is often interested in its Hirzebruch $\chi_{y}$ genus [24], which is defined by

$$
\chi_{y}(X)=\sum_{p \in \mathbb{Z}_{+} \cup\{0\}}^{d}(-y)^{p} \sum_{k \in \mathbb{Z}_{+} \cup\{0\}}^{d}(-1)^{k} \operatorname{dim} H^{k}\left(X, \Lambda^{p}(\overline{T X})\right) \stackrel{(\text { by HRR })}{=} \int_{X} \prod_{j \in \mathbb{Z}_{+}} \frac{x_{j}\left(1-y e^{-x_{j}}\right)}{\left(1-e^{-x_{j}}\right)} .
$$

The $\chi_{y}$-genus reduces to other invariants for special values of $y$ :

- Suppose, as before, that $X$ is a compact complex manifold, and $\pi: E \rightarrow X$ an holomorphic vector bundle on $X$. The Riemann-Roch number of $E$ is defined by

$$
\chi(X, E)=\sum_{k \in \mathbb{Z}_{+} \cup\{0\}}(-1)^{k} \operatorname{dim} H^{k}\left(X, \mathscr{O}_{X}(E)\right),
$$

where $\mathscr{O}_{X}(E)$ is the sheaf of holomorphic section to $E$, and $H^{k}\left(X, \mathscr{O}_{X}(E)\right)$ is the $k$-th cohomology of $\mathscr{O}_{X}(E)$. It has the following property: If $0 \rightarrow E_{1} \rightarrow E_{2} \rightarrow \cdots \rightarrow E_{n} \rightarrow 0$ is an exact sequence of holomorphic vector bundles on $X$, then we have

$$
\chi\left(X, E_{1}\right)-\chi\left(X, E_{2}\right)-\cdots+\chi\left(X, E_{n}\right)=0 .
$$

Hence, if one denotes by $K(X)$ the Grothendieck ring of holomorphic vector bundles over $X$, then one gets an additive homomorphism $\chi(X, \bullet): K(X) \rightarrow \mathbb{Z}$. It is straightforward to 
extend $\chi(X, \bullet)$ to formal power series with coefficients in $K(X)$. The Riemann-Roch number of $E$ can be computed by the HRR Theorem, as follows. Let $x_{1}, \ldots, x_{d}$ and $z_{1}, \ldots, z_{r}$ be the formal Chern roots of $T X$ and $E$, respectively, then

$$
\chi(X, E)=\int_{X} \operatorname{ch}(E) \operatorname{Td}(T X)=\int_{X} \sum_{k=1}^{r} e^{z_{k}} \prod_{j} \frac{x_{j}}{1-e^{-x_{j}}} .
$$

- The arithmetic genus of an irreducible, projective curve $\mathscr{C}$ is $\operatorname{dim} H^{1}(\mathscr{C}, \mathscr{O})$, where $\mathscr{O}$ is the structure sheaf of holomorphic functions on $\mathscr{C}$. For a smooth curve, this is the same as the geometric genus, $\chi_{y}(X)=\sum_{k \in \mathbb{Z}_{+} \cup\{0\}}^{d}(-1)^{k} \operatorname{dim} H^{k}\left(X, \mathscr{O}_{X}\right)$; however, unlike the geometric genus, the arithmetic genus has the nice feature that it remains constant in families of curves with possibly singular fibers. Intuitively, this means that the arithmetic genus of a nodal curve is the geometric genus of the curve obtained by smoothing out the nodes.

\section{Elliptic genera of hypersurfaces and complete intersections}

Let $X$ be a finite dimensional $\mathbb{Z}$-graded complex vector space. Consider the formal power series of vector spaces

$$
\mathscr{E} l l(X ; q, y)=y^{-\operatorname{dim} X / 2} \bigotimes_{n \in \mathbb{Z}_{+}}\left(\Lambda_{y q^{n-1}}(\bar{X}) \otimes \Lambda_{q^{n-1}}(X) \otimes S_{q^{n}}(\bar{X}) \otimes S_{q^{n}}(X)\right) .
$$

Suppose $\mathscr{E} l l(T X ; q, y)=\sum_{k, \ell} y^{k} q^{\ell} \mathscr{E}_{k \ell}$, then the graded space $\mathscr{E} l l(X)=\sum_{k, \ell} \mathscr{E}_{k \ell}$ has a structure of an $N=2$ superconformal vertex algebra. It is straightforward to see that the operation $\mathscr{E} l l$ is multiplicative in the following sense:

$$
\mathscr{E} l l\left(X \oplus X^{\prime} ; q, y\right)=\mathscr{E} l l(X ; q, y) \otimes \mathscr{E} l l\left(X^{\prime} ; q, y\right) .
$$

Elliptic genera of hypersurfaces. Let $X$ be a complex manifold and let $Y \subset X$ be a smooth hypersurface. Denote by $[Y]$ the line bundle on $X$ associated to the divisor $Y$. The adjunction formula states that $\left.N_{Y / X} \cong[Y]\right|_{Y}$, where $N_{Y / X}=\left.T X\right|_{Y} / T Y$ is the normal bundle of $Y$ in $X$. The exact sequence

$$
\begin{aligned}
& \left.0 \longrightarrow T Y \longrightarrow T X\right|_{Y} \longrightarrow N_{Y / X} \rightarrow 0 \quad \text { can be rewritten as } \\
& \left.\left.0 \longrightarrow T Y \longrightarrow T X\right|_{Y} \longrightarrow[Y]\right|_{Y} \longrightarrow 0
\end{aligned}
$$

Note that, in $K$-theory, $T Y=\left.(T X-[Y])\right|_{Y}$ (the later can be referred to as the virtual tangent bundle of $Y$ in $X)$. The following statement holds [25]: Suppose $Y$ is a smooth hypersurface of a compact complex manifold $X$, then ${ }^{1}$

$$
\chi_{-y}(Y)=\chi\left(X, \Lambda_{-y}(T X)(1 \ominus y[-Y])^{-1}(1 \ominus[-Y])\right) .
$$

\footnotetext{
${ }^{1}$ Note that $c(T Y)=\left.c(T X) c([Y])^{-1}\right|_{Y}$. Let us introduce the following notation: $c(E)=\prod_{j} c\left(E_{j}\right)^{(-1)^{j}}$ for a $\mathbb{Z}$ graded vector bundle $E=\bigoplus_{j} E_{j}$. We have $c(T Y)=\left.c(T X \ominus[Y])\right|_{Y}$, and $\chi(Y)=\int_{Y} c(T Y)=\int_{X} c(T Y) c_{1}\left(N_{Y / X}\right)=$ $\int_{X} c(T X \ominus[Y]) c_{1}([Y])$, where $c_{1}([Y])$ is the first Chern class of $[Y]$.
} 
In terms of formal Chern roots $\left\{x_{j}\right\}$ we have

$$
\begin{aligned}
\chi_{-y}(Y) \stackrel{\text { (by HRR })}{=} y^{-\operatorname{dim} Y / 2} \int_{X} \prod_{n \in \mathbb{Z}_{+}} \frac{\left(1-q^{n-1} e^{-c_{1}}\right)\left(1-q^{n} e^{c_{1}}\right)}{\left(1-y q^{n-1} e^{-c_{1}}\right)\left(1-y^{-1} q^{n} e^{c_{1}}\right)} \\
\times \prod_{j=1}^{\operatorname{dim} X} x_{j} \prod_{n \in \mathbb{Z}_{+}} \frac{\left(1-y q^{n-1} e^{-x_{j}}\right)\left(1-y^{-1} q^{n} e^{x_{j}}\right)}{\left(1-q^{n-1} e^{-x_{j}}\right)\left(1-q^{n} e^{x_{j}}\right)} . \\
=\quad y^{-\operatorname{dim} Y / 2} \int_{X^{\mathbb{C}}}\left[\frac{\mathscr{R}(s=-\widehat{c}(1-i t))}{\mathscr{R}(s=(w-\widehat{c})(1-i t))}\right]\left[\frac{\mathscr{R}(s=(1+\widehat{c})(1-i t))}{\mathscr{R}(s=(1-w+\widehat{c})(1-i t))}\right] \\
\times \prod_{j=1}^{\operatorname{dim} X} x_{j}\left[\frac{\mathscr{R}\left(s=\left(w-\widehat{x}_{j}\right)(1-i t)\right)}{\mathscr{R}\left(s=-\widehat{x}_{j}(1-i t)\right)}\right]\left[\frac{\mathscr{R}\left(s=\left(1-w+\widehat{x}_{j}\right)(1-i t)\right)}{\mathscr{R}\left(s=\left(1+\widehat{x}_{j}\right)(1-i t)\right)}\right],
\end{aligned}
$$

where $c_{1}=\widehat{c} / 2 \pi i$. If $q=0$, one has $\mathscr{E} l l(T X ; 0, y)=y^{-\operatorname{dim} X / 2} \Lambda_{-y}(T X)$ and $\mathscr{E} l l([Y] ; 0, y)=y^{-1 / 2}(1-$ $y[-Y])$. For a smooth hyperface $Y$ of a compact complex manifold $X$, we have [25]: $\chi_{-y}(Y)=$ $\chi\left(X, \Lambda_{-y}(T X)(1 \ominus y[-Y])^{-1}(1 \ominus[-Y])\right)$. And, in terms of formal Chern roots, we finally obtain

$$
\chi_{-y}(Y)=\int_{X} \frac{1-e^{-c_{1}}}{1-y e^{-c_{1}}} \prod_{j=1}^{\operatorname{dim} X} \frac{x_{j}\left(1-y e^{-x_{j}}\right)}{\left(1-e^{-x_{j}}\right)} .
$$

Smooth complete intersections. Assume now that $\pi: V \rightarrow X$ is an holomorphic vector bundle on $X$ of rank $r, s: X \rightarrow V$ an holomorphic section transverse to the zero section, and $Y:=s^{-1}(0)$ is a complex submanifold of $X$, with $\left.N_{Y / X} \cong V\right|_{Y}$. From the exact sequence $\left.0 \rightarrow T Y \rightarrow T X\right|_{Y} \rightarrow$ $N_{Y / X} \rightarrow 0$ by the multiplicative property of $\mathscr{E} l l$, it follows that [25]

$$
\begin{aligned}
& \mathscr{E} l l(T Y ; q, y) \mathscr{E} l l\left(\left.V\right|_{Y} ; q ; y\right)=\mathscr{E} l l\left(\left.T X\right|_{Y} ; q, y\right)=\left.\mathscr{E} l l(T X ; q, y)\right|_{Y}, \\
& \mathscr{E} l l(T Y ; q, y)=\left.\mathscr{E} l l(T X ; q, y) \mathscr{E} l l(V ; q ; y)^{-1}\right|_{Y} \Longrightarrow \\
& \chi(Y, \mathscr{E} l l(T Y ; q, y))=\chi\left(Y,\left.\mathscr{E} l l(T X \ominus V ; q, y)\right|_{Y}\right) .
\end{aligned}
$$

Tensoring the Koszul complex [26]

$$
0 \longrightarrow \mathscr{O}_{X}\left(\Lambda^{r}(\bar{V})\right) \stackrel{i_{s}}{\longrightarrow} \mathscr{O}_{X}\left(\Lambda^{r-1}(\bar{V})\right) \stackrel{i_{s}}{\longrightarrow} \cdots \stackrel{i_{s}}{\longrightarrow} \mathscr{O}_{X}\left(\Lambda^{1}(\bar{V})\right) \stackrel{i_{s}}{\longrightarrow} \mathscr{O}_{X} \longrightarrow \mathscr{O}_{Y} \longrightarrow 0
$$

by $\mathscr{E} l l(T X \ominus V ; q, y)$, we get the exact sequence

$$
\begin{aligned}
0 & \longrightarrow \mathscr{O}_{X}\left(\mathscr{E} l l(T X \ominus V ; q, y) \Lambda^{r} \bar{V}\right) \stackrel{i_{s}}{\longrightarrow} \mathscr{O}_{X}\left(\mathscr{E} l l(T X \ominus V ; q, y) \Lambda^{r-1}(\bar{V})\right) \stackrel{i_{s}}{\longrightarrow} \cdots \\
& \stackrel{i_{s}}{\longrightarrow} \mathscr{O}_{X}\left(\mathscr{E} l l(T X \ominus V ; q, y) \Lambda^{-1}(\bar{V}) \stackrel{i_{s}}{\longrightarrow} \mathscr{O}_{X}(\mathscr{E} l l(T X \ominus V ; q, y))\right. \\
& \left.\longrightarrow \mathscr{O}_{X}(\mathscr{E} l l(T X \ominus V ; q, y))\right|_{Y} \longrightarrow 0,
\end{aligned}
$$

Suppose now that $Y$ is the zero set of an holomorphic section to an holomorphic vector bundle $V$ on a compact complex manifold $X$, which is transverse to the zero section. Then, by taking the Riemann-Roch number, one gets [25]:

$$
\chi(Y ; q, y)=\chi\left(Y,\left.\mathscr{E} l l(T X \ominus V ; q, y)\right|_{Y}\right)=\chi\left(X, \mathscr{E} l l(T X \ominus V ; q, y) \Lambda_{-1}(\bar{V})\right)
$$


ASs before denote by $\left\{z_{k}\right\}_{i=1}^{r}$ the formal Chern roots of $V$. Then, we have

$$
\begin{aligned}
\chi(Y ; q, y) & =y^{-\operatorname{dim} Y / 2} \int_{X} \prod_{k=1}^{r} \prod_{n \in \mathbb{Z}_{+}} \frac{\left(1-q^{n-1} e^{-z_{k}}\right)\left(1-q^{n} e^{z_{k}}\right)}{\left(1-y q^{n-1} e^{-z_{k}}\right)\left(1-y^{-1} q^{n} e^{\left.z_{k}\right)}\right.} \\
& \times \prod_{j=1}^{\operatorname{dim} X} x_{j} \prod_{n \in \mathbb{Z}_{+}} \frac{\left(1-y q^{n-1} e^{-x_{j}}\right)\left(1-y^{-1} q^{n} e^{x_{j}}\right)}{\left(1-q^{n-1} e^{-x_{j}}\right)\left(1-q^{n} e^{x_{j}}\right)} \\
& =y^{-\operatorname{dim} Y / 2} \int_{X} \prod_{k=1}^{r}\left[\frac{\mathscr{R}\left(s=-\widehat{z}_{k}(1-i t)\right)}{\mathscr{R}\left(s=\left(w-\widehat{z}_{k}\right)(1-i t)\right)}\right]\left[\frac{\mathscr{R}\left(s=\left(1+\widehat{z}_{k}\right)(1-i t)\right)}{\mathscr{R}\left(s=\left(1-w+\widehat{z}_{k}\right)(1-i t)\right)}\right] \\
& \times \prod_{j=1}^{\operatorname{dim} X} x_{j}\left[\frac{\mathscr{R}\left(s=\left(w-\widehat{x}_{j}\right)(1-i t)\right)}{\mathscr{R}\left(s=-\widehat{x}_{j}(1-i t)\right)}\right]\left[\frac{\mathscr{R}\left(s=\left(1-w+\widehat{x}_{j}\right)(1-i t)\right)}{\mathscr{R}\left(s=\left(1+\widehat{x}_{j}\right)(1-i t)\right)}\right],
\end{aligned}
$$

where $\widehat{z}_{k}=z_{k} / 2 \pi i$. The last products in integrands (5.4) and (5.7) are the same, both have been calculated in terms of formal Chern roots of $T X$. (Recall that the first product in integrand (5.7)) is given in terms of formal Chern roots of vector bundle $V$ on $X$.) If $q=0$ then we get back a similar to result to (5.5):

$$
\chi_{-y}(Y)=\chi\left(X, \Lambda_{-y}(T X)\left(\Lambda_{-y} \bar{V}\right)^{-1} \Lambda_{-1} \bar{V}\right)=\int_{X} \prod_{k=1}^{r} \frac{\left(1-e^{-z_{k}}\right)}{\left(1-y e^{-z_{k}}\right)} \prod_{j=1}^{\operatorname{dim} X} \frac{x_{j}\left(1-y e^{-x_{j}}\right)}{\left(1-e^{-x_{j}}\right)} .
$$

\section{Conclusions}

In this paper we have discussed how the spectral functions of an $A d S_{3}$-asymptotic geometry are intertwined with the Casimir effect in topological field theory, quantum gravity and elliptic genera of an hypersurface and complete intersections. It is of course of utmost importance to find generalizations of the examples considered here. Perhaps one could make analogues of $\mathrm{N}$ fold products for other type of supergravity solutions, the Hilbert modular variety, for example $[27,5]$. The Hilbert modular variety carries a natural structure as a quasi-projective variety and its cohomology groups inherit a Hodge structure. In fact the Hilbert modular group is a simplified example of the cohomology theory of arithmetic groups and it is the only special case in which the cohomology can be determined explicitly.

Having advocated in this paper the basic role of cohomologies of infinite-dimensional Lie algebras, we are now naturally led to other problems, related to the quantization of nonlinear sigma models and gravity and the Casimir effect for the quantized models. One might ask whether the quantum behaviours of these models can be algebraically interpreted by means of infinitesimal deformations of the corresponding Lie algebra. No doubt this analysis requires a new degree of mathematical sophistication. Perhaps all the concepts of what should be the "deformation theory of everything" might be tested in the case of associative algebras, which are algebras over operads [28]. In many examples dealing with algebras over operads, specific arguments on the universality of associative algebras are called forth. This may suggest that a connection between the Casimir effect for deformation theory and algebras over operads could indeed exist.

\section{Acknowledgements}

$\mathrm{AAB}$ would like to acknowledge the Conselho Nacional de Desenvolvimento Científico e Tecnológico (CNPq, Brazil), Fundação Araucária (Paraná, Brazil) and the ESF Research Network 
CASIMIR for financial support. EE's research has been partly supported by MICINN (Spain), contract PR2011-0128 and project FIS2010-15640, by the CPAN Consolider Ingenio Project, and by AGAUR (Generalitat de Catalunya), contract 2009SGR-994, and his research was partly carried out while on leave at the Department of Physics and Astronomy, Dartmouth College, NH, USA. RML would like to acknowledge Coordenação de Aperfeiçoamento de Pessoal de Nível Superior (CAPES, Brazil) and Fundação Araucária (Paraná, Brazil) for financial support.

\section{References}

[1] T. Eguchi and M. Jinzenji, Generalization of Calabi-Yau/Landau-Ginzburg correspondence, JHEP 02 (2000) 028 [hep-th/9911220].

[2] E. Elizalde, M. Bordag and K. Kirsten, Casimir energy for a massive fermionic quantum field with a spherical boundary, J. Phys. A31 (1998) 1743-1759 [hep-th/9707083].

[3] M. Bordag, G. L. Klimchitskaya, U. Mohideen and V. M. Mostepanenko, Advances in the Casimir Effect, Oxford University Press Inc., 2009.

[4] A. Strominger and C. Vafa, Microscopic origin of Bekenstein-Hawking entropy, Phys. Lett. B 379 (1996) 99-104 [hep-th/9601029].

[5] L. Bonora and A. A. Bytsenko, Partition functions for quantum gravity, black holes, elliptic genera and Lie algebra homologies, Nucl. Phys. B 852 (2011) 508-537 [hep-th/1105.4571].

[6] A. Maloney and E. Witten, Quantum Gravity Partition Function In Three Dimensions, JHEP 1002 (2010) 029 [hep-th/0712.0155].

[7] L. Bonora, A. A. Bytsenko and E. Elizalde, String partition functions, Hilbert schemes and affine Lie algebra representations on homology groups, J. Phys. A: Math. Theor. 45 (2012) 374002 (41pp) [hep-th/1206.0664].

[8] P. Perry and F. Williams, Selberg zeta function and trace formula for the BTZ black hole, Internat. J. of Pure and Applied Math. 9 (2003) 1-21.

[9] A. A. Belavin, A. M. Polyakov and A. B. Zamolodchikov, Infinite conformal symmetry of critical fluctuations in two dimensions, J. Stat. Phys. 34 (1984) 763-774.

[10] A. A. Belavin, A. M. Polyakov and A. B. Zamolodchikov, Infinite conformal symmetry in two-dimensional quantum field theory, Nucl. Phys. B 241 (1984) 333-380.

[11] V. G. Kac, Infinite Dimensional Lie algebras, (3rd Ed.), Cambridge Univ. Press, 1990.

[12] A. A. Bytsenko and M. E. X. Guimãraes, Truncated Heat Kernel and One-Loop Determinants for the BTZ Geometry, Eur. Phys. J. C 58 (2008) 511-516 [hep-th/0809.1416].

[13] A. A. Bytsenko and M. E. X. Guimãraes, Partition Functions of Three-Dimensional Quantum Gravity and the Black Hole Entropy, J. Phys. Conf. Ser. 161 (2009) 012023 [hep-th/0807.2222].

[14] R. Dijkgraaf, Discrete torsion and symmetric products, hep-th/9912101.

[15] M. Kleban, M. Porrati and R. Rabadan, Poincaré Recurrences and Topological Diversity, JHEP 0410 (2004) 030 [hep-th/0407192].

[16] J. Manschot, $A d S_{3}$ Partition Functions Reconstructed, JHEP 0710 (2007) 103 [hep-th/0707.1159].

[17] H. Iwaniec, Spectral Methods of Authomorphic Forms, (Graduate Studies in Mathematics, 53), Amer. Math. Soc. 2002. 
[18] E. Elizalde, S.D. Odintsov, and A.A. Saharian, Repulsive Casimir effect from extra dimensions and Robin boundary conditions: from branes to pistons, Physical Review D79 (2009) 065023 [hep-th/0902.0717].

[19] T. Eguchi, H. Ooguri, A. Taormina and S.-K. Yang, Superconformal algebras and string compactification on manifold with $S U(N)$ holonomy, Nucl. Phys. B 315 (1989) 193-221.

[20] E. Witten, On Landau-Ginzburg description of N=2 minimal models, Int. J. Mod. Phys. A 9 (1994) 4783-4800.

[21] T. Kawai, Y. Yamada and S.-K. Yang, Elliptic Genera and N=2 Superconformal Field Theory, Nucl. Phys. B 414 (1994) 191-212 [hep-th/9396996].

[22] R. Dijkgraaf, G. Moore, E. Verlinde and H. Verlinde, Elliptic Genera of Symmetric Products and Second Quantized Strings, Commun. Math. Phys. 185 (1997) 197-209 [hep-th/9608096].

[23] L.A. Borisov and A. Libgober, Elliptic Genera of Toric Varieties and Applications to Mirror Symmetry, Invent. Math. 140 (2000) 453-485.

[24] J. Li, K. Liu and J. Zhou, Topological String Partition Functions as Equivariant Indices, Asian J. Math. 10 (2006) 81-114 [math/0412089].

[25] X. Ma and J. Zhou, Elliptic genera of complete intersections in weighted projective spaces, Int. J. Math. 22 (2011) 695-712 [math.AG/0411081].

[26] P. Griffiths, J. Harris, Principles of algebraic geometry. Pure and Applied Mathematics, Wiley-Interscience [John Wiley \& Sons], New York, 1978.

[27] A.A. Bytsenko and E. Elizalde, AdS Solutions in Gauge Supergravities and the Global Anomaly for the Product of Complex Two-Cycles, Eur. Phys. J. C 71 (2011) 1592-1597 [hep-th/1105.0309].

[28] M. Kontsevich and Y. Soibelman, Deformations of algebras over operads and the Deligne conjecture, Conférence Moshé Flato 1999, Vol. I (Dijon), 255-307, Math. Phys. Stud., 21, Kluwer Acad. Publ., Dordrecht, 2000 [math/0001151]. 